\title{
The effect of pre- operative topical flurbiprofen or diclofenac on pupil dilatation
}

\begin{abstract}
Purpose To assess the clinical benefit and relative efficacy of pre-operative diclofenac and flurbiprofen drops in routine cataract surgery.

Methods Fifty-two patients undergoing extracapsular cataract extraction with lens implantation were randomised in a doublemasked study to compare the efficacy of diclofenac, flurbiprofen and placebo drops in maintaining per-operative mydriasis and reducing post-operative inflammation. Balanced salt solution containing adrenaline was used in all patients. Pupil size was measured prior to the corneal section and after the completion of the operation. The degree of pain, redness, flare and cells in the anterior chamber and intraocular pressure were recorded on the day after surgery. The three groups were analysed with respect to change in pupil size, intraocular pressure and degree of inflammation.

Results The change in pupil size was significantly different among the three groups ( $p=0.01$ ), there being a smaller decrease in the treatment groups compared with the placebo group and in the diclofenac treatment group compared with the flurbiprofen treatment group. Significantly less post-operative redness was recorded in the diclofenac treatment group compared with the other groups $(p=0.001)$. No significant difference was found between the groups as regards anterior chamber cells, flare or intraocular pressure change.

Conclusions Pre-operative diclofenac and flurbiprofen drops are effective in maintaining intraoperative mydriasis.

Diclofenac reduces post-operative redness on day 1 . These effects are of debatable clinical benefit.
\end{abstract}

V.T. Thaller

Royal Eye Infirmary

Plymouth, UK

M.K. Kulshrestha

Birmingham and Midland

Eye Centre

Birmingham, UK

\section{K. Bell}

Royal Victorian Eye and Ear Hospital

Melbourne, Australia

Mr Manoj Kulshrestha Birmingham and Midland Eye Centre

City Hospital NHS Trust

Western Road

Birmingham B18 7QU, UK

Tel: + $44(0) 17899823508$

Fax: +44 (0)1212493734

e-mail:

manojkulshrestha@excite.co.uk.

Received: 24 February 1997 Accepted in revised form: 19 January 2000
Key words Cataract, Diclofenac, Flurbiprofen, Mydriasis, Pre-operative, Pupil size
V.T. THALLER, M.K. KULSHRESTHA, K. BELL
The maintenance of mydriasis is important during cataract surgery to facilitate uncomplicated cortex removal and intraocular lens insertion. ${ }^{1,2}$ The administration of preoperative topical non-steroidal antiinflammatory drops (NSAIDs) ${ }^{3-12}$ and the use of adrenaline in the irrigating solution ${ }^{4,6,13,14}$ are both effective at maintaining mydriasis. An additive effect on the maintenance of pupil size has been demonstrated when both methods are used together. ${ }^{4,6,15,16}$ However, this effect has been small. This study attempted to quantify this effect and to determine whether flurbiprofen or diclofenac is the more effective preparation.

In addition it has been our impression that pre-operative NSAIDs give whiter eyes postoperatively. We therefore looked for a clinically observable difference in post-operative inflammation on the first post-operative day.

\section{Methods}

A randomised, double-masked, placebocontrolled method was used with two active treatment groups and a placebo group.

Consecutive patients listed for elective extracapsular cataract extraction with intraocular lens implantation were invited to take part in the study. Local research ethics committee approval was obtained, as was informed written consent from all patients entering the trial. Stringent exclusion criteria were employed in patient selection to minimise the possible confounding effects of concurrent medication or previous eye treatment (Table 1 )

Fifty-two patients were randomly assigned to either the control group or one of two treatment groups. All patients received routine pre-operative dilating drops consisting of 4 drops each of cyclopentolate $1 \%$ and phenylephrine $10 \%$. In addition group A received placebo drops (diclofenac vehicle solution was used), group B received diclofenac sodium $0.1 \%$ and group $C$ received flurbiprofen 
Table 1. Exclusion criteria applied in the selection of study participants

Age $<18$ years

Pregnancy or lactation

Known hypersensitivity to diclofenac, flurbiprofen or other NSAIDs

Concurrent or recent (within the two preceding weeks) medication with systemic steroids or NSAIDs, cytotoxic or other immunosuppressant agents

Concurrent medication with systemic drugs with autonomic side effects

Previous ocular surgery or serious injury in the operated eye

History or signs of recurrent uveitis and/or evidence of iris sphincter atrophy

Use of any topical ophthalmic medications in the preceding two weeks apart from ocular lubricants or prophylactic topical antibiotics Participating in another trial

Non-compliance with protocol

Glaucoma patients

sodium $0.03 \%$. One drop was given every 30 min starting $2 \mathrm{~h}$ before surgery for a total of four doses of the stated agent in each group.

All surgery was performed by the same surgeon using a standard extracapsular cataract technique with a corneal section under a general or local anaesthetic. Sodium hyaluronate (aspirated at the end of the procedure) was used as was a balanced salt solution containing adrenaline, at a concentration of 1 part per million, for irrigating the anterior chamber.

Pupillary diameter was recorded in millimetres using a caliper by the surgeon prior to the corneal section and at the end of the operation following reformation of the anterior chamber.

The degree of surgical iris trauma was graded as mild $(=1)$, moderate $(=2)$ or severe $(=3)$. Patients with severe complications such as vitreous loss, or with complications such as circumlimbal subconjunctival haemorrhage which would prevent post-operative assessment of conjunctival redness, were excluded from analysis.

Table 2. The clinical grading scales employed to assess post-operative pain and inflammation in 52 cataract patients

\begin{tabular}{lcl}
\hline Category & Scale & Criteria \\
\hline Post-operative pain & 0 & None \\
& 1 & Mild \\
& 2 & Moderate or constant \\
& 3 & Severe/analgesia required \\
Redness & 0 & Absent \\
(bulbar injection) & & \\
& 1 & Mild \\
& 2 & Moderate/circumlimbal \\
& 3 & Severe/whole bulbar conjunctiva \\
Flare & 0 & None \\
& 1 & Barely detectable \\
& 2 & Mild \\
& 3 & Moderate \\
& 4 & Strong \\
& 5 & Severe/coagulated aqueous \\
& 0 & None \\
Cells & 1 & Occasional \\
& 2 & Few \\
& 3 & Moderate \\
& 4 & Many \\
& 5 & Hypopyon \\
\hline
\end{tabular}

On the first post-operative day, the degree of pain, redness, flare and cells in the anterior chamber were assessed according to set grading scales (Table 2).

The intraocular pressure was recorded preoperatively (within 1 month of surgery) and of the first post-operative day.

An analysis of variance (ANOVA) test was used to compare pupil size and intraocular pressure results among the three groups. A Kruskal-Wallis analysis of variance was used on the ordinal data recorded from the graded scales. A $p$ value of less than 0.05 was considered to be statistically significant.

\section{Results}

Fifteen patients were assigned to the placebo group, 17 to the flurbiprofen group and 20 to the diclofenac group. Only 2 patients in the study had general anaesthesia: 1 in the placebo and 1 in the diclofenac group. All other patients had a local anaesthetic.

No statistically significant difference was noted among the groups in the pre-operative intraocular pressure measurement $(p=0.79)$, the post-operative intraocular pressure $(p=0.29)$, or in the pre- to postoperative change in intraocular pressure $(p=0.39)$. There was no statistically significant difference in degree of iris trauma among the groups $(p=0.79)$.

Pupil size in the three treatment groups preoperatively was comparable $(p=0.79)$. Change in pupil size from pre-operative to post-operative measurements was statistically significant among the treatment groups $(p=0.01)$ (Table 3). A follow-up analysis using least significant difference showed that the change in pupil size was significantly smaller in both the NSAID groups compared with the placebo group and also in the diclofenac treatment group as compared with the flurbiprofen treatment group.

There was a statistically significant difference in the degree of redness post-operatively among the three groups ( $p=0.001$ ). A follow-up analysis using least significant difference showed diclofenac caused a statistically significant reduction in the degree of redness post-operatively compared with flurbiprofen and placebo. Flurbiprofen caused no significant difference in redness compared with placebo drops. 


\begin{tabular}{lccc}
\hline & Placebo & Flurbiprofen & Diclofenac \\
\hline No. of patients & 15 & 17 & 20 \\
Mean pre-operative pupil size $(\mathrm{mm})$ & 8.00 & 8.15 & 7.98 \\
Mean post-operative pupil size $(\mathrm{mm})$ & 7.47 & 7.94 & 7.93 \\
Mean change in pupil size $(\mathrm{mm})$ & -0.53 & -0.21 & -0.05 \\
\hline
\end{tabular}

There was no clinically significant difference among the three groups in the flare $(p=0.51)$, cells $(p=0.53)$ or pain $(p=0.07)$ levels recorded post-operatively. We found no adverse effects from either drop other than mild stinging on instillation.

\section{Discussion}

It is now well established that NSAIDs reduce intraoperative miosis during cataract surgery. ${ }^{3-12}$ This is confirmed in this study. Of the two NSAIDs, diclofenac was found to be more effective than flurbiprofen in producing this effect (Table 3).

Several studies have shown that adrenaline is more effective in maintaining intraoperative mydriasis than pre-operative treatment with NSAIDs, ${ }^{4,6}$ which may indicate that there is little to gain from using NSAIDs in place of adrenaline in the anterior chamber irrigation fluid. Adrenaline is also more convenient, as the recommended pre-operative treatment regime for NSAIDs is 4 drops over $2 \mathrm{~h}$ while our routine preoperative mydriatics are administered over $1 \mathrm{~h}$, so that patients requiring NSAIDs must be admitted an hour sooner than they might otherwise be.

We found a statistically significant reduction in the degree of redness on the first post-operative day in eyes pre-treated with diclofenac. This bears out our clinical impression prior to commencing this study.

Our study showed no statistically significant difference in the flare and cells in the anterior chamber post-operatively, nor in post-operative pain. Therefore the benefit of this reduction in redness is isolated and may not be clinically significant.

It could be argued that measurement with a laser cell/ flare meter might have been a more sensitive and accurate way of assessing inflammation. Nevertheless, a study has shown close correlation between laser flare and cell measurements and clinical assessments following cataract surgery. ${ }^{17}$ Our purpose was to demonstrate whether there was a clinically significant anti-inflammatory effect from NSAIDs, using a method which we use in everyday practice. For this we consider grading by slit-lamp microscopy to be adequate. Blaydes et al. ${ }^{18}$ also found no significant difference (using clinical slit-lamp grading) in the day 1 post-operative anterior chamber inflammation between patients undergoing phacoemulsification cataract extraction and receiving either NSAIDs or placebo drops pre-operatively.

Our findings relate specifically to a standard surgical technique. The post-operative intraocular pressure rise was not influenced by the trial drops. This agrees with the findings of Strelow et al. ${ }^{19}$ Arguably corneal incisions tend to cause less redness, inflammation and pressure. Therefore extrapolation of our results to limbal sections or phacoemulsification surgery should be done with caution.

In our attempt to exclude confounding factors, such as diseases or treatments known to affect the autonomic control of pupil function or post-operative inflammation, we were surprised to find a high exclusion rate approaching $40 \%$ of patients listed for cataract surgery. A surprising number of patients are already on oral NSAIDs. It is likely that without these exclusions the clinical significance of the differences which we have been able to demonstrate would be even smaller.

The additional cost of the routine use of NSAIDs is about $£ 4$ per patient. Whilst not high, this would add an annual cost of $£ 1200$ for a surgeon doing 300 cataract operations a year.

More recently there has been evidence that ketorolac and flurbiprofen may have a role in preventing pseudophakic cystoid macular oedema. ${ }^{20,21}$ The efficacy of diclofenac has not been tested in this role to our knowledge by a masked prospective study. It is known that indomethacin inhibits lens epithelial cell proliferation in vitro, ${ }^{22}$ though whether the use of NSAIDs affects the YAG capsulotomy rate following cataract surgery is unknown.

In conclusion, this study provides little support for the routine use of pre-operative diclofenac or flurbiprofen drops in extracapsular cataract surgery, as the effect of these drugs, though statistically significant in some parameters, was small and probably of little clinical benefit. Diclofenac was the more effective agent in maintaining mydriasis.

The intraocular forces acting on the pupil during phacoemulsification are different. Maintaining pupil size during phacoemulsification is important for sculpting of the lens nucleus and subsequent cortex aspiration. Further masked prospective studies are required to assess the usefulness of NSAIDs during this surgical procedure. There is also a need for more clinical investigations to ascertain whether these topical preparations are useful in the prevention of postoperative cystoid macular oedema and posterior capsule opacification.

\section{References}

1. Goodman DF, Stark WJ, Gottsch ID. Complications of cataract extraction with intraocular lens implantation. Ophthalmic Surg 1989;20:132-40.

2. Williams HP, Manipulation of the iris in extracapsular lens extraction. Trans Ophthalmol Soc UK 1985;104:553-7. 
3. Allaire C, Lablache Combier M, Trinquand C, Bazin R. Comparative efficacy of $0.1 \%$ indomethacin eyedrops, $0.03 \%$ flurbiprofen eyedrops and placebo for maintaining peroperative mydriasis. J Fr Ophtalmol 1994;17:103-9.

4. Cillino S, Casanova F, Cucco F, Ponte F. Topical flurbiprofen in extracapsular cataract surgery: effect on pupillary diameter and iris fluorescein leakage. J Cataract Refract Surg 1993;19:622-5.

5. Drews RC, Katsev DA. Ocufen and pupillary dilation during cataract surgery. J Cataract Refract Surg 1989;15:445-8.

6. Gimbel HV. The effect of treatment with topical nonsteroidal anti-inflammatory drugs with and without intraoperative epinephrine on the maintenance of mydriasis during cataract surgery. Ophthalmology 1989;96:585-8.

7. Keates RH, McGowan IKA. Clinical trial of flurbiprofen to maintain pupillary dilation during cataract surgery. Ann Ophthalmol 1984;16:919-21.

8. Keates RH, McGowan KA. The effect of topical indomethocin ophthalmic solution in maintaining mydriasis during cataract surgery. Ann Ophthalmol 1984;16:1116-21.

9. Keulen-De Vos HCJ, Van Rij G, Renardel De Lavalette JCG, Jansen JTG. Effect of indomethacin in preventing surgically induced miosis. Br J Ophthalmol 1983;67:94-6.

10. Psilas K, Kalogeropoidos C, Locatzicos E, Asproudis I, Petroutsos O. The effect of indomethacin, diclofenac and flurbiprofen on the maintenance of mydriasis during extracapsular cataract extraction. Doc Ophthalmol 1992;81:292-300.

11. Sawa M, Masuda K. Topical indomethacin in soft cataract aspiration. Jpn J Ophthalmol 1976;20:514-9.

12. Stark WJ, Fagadau WR, Stewart BR, Crandell AS, deFaller SK, Reaves TA, Edwards Klein P. Reduction of papillary constriction during cataract surgery using suprofen. Arch Ophthalmol 1986;104:364-6.

13. Elliott A, Carter C. Pupil size after extracapsular cataract extraction and posterior chamber lens implantation: a prospective randomised trial of epinephrine and acetylcholine. Ophthalmic Surg 1989;20:591-4.
14. Corben $\mathrm{MC}$, Richards $\mathrm{AB}$. Intraocular adrenaline maintains mydriasis during cataract surgery. Br J Ophthalmol 1994;78:95-8

15. Heinrichs DA, Leith AB. Effect of flurbiprofen on the maintenance of pupillary dilatation during cataract surgery. Can J Ophthalmol 1990;25:239-42.

16. Sachdev MS, Gupta SK, Singh K, Dada VK, Talwar D. Comparative efficacy of diclofenac and flurbiprofen for the maintenance of pupillary dilatation during cataract surgery. Ophthalmic Surg 1994;25:92-4.

17. el-Maghraby A, Marzouki A, Matheen TM, Souchek J, Van der Karr M. Reproducibility and validity of laser flare/cell meter measurements of intraocular inflammation. J Cataract Refract Surg 1993;19:52-5.

18. Blaydes JE, Kelley EP, Wait JG, DeGryse RE, Harper DG, Novack GD. Flurbiprofen $0.03 \%$ for the control of inflammation following cataract extraction by phacoemulsification. J Cataract Refract Surg 1993;19:481-7.

19. Strelow SA, Driebe WT, Sherwood NB, Guy JR, Broncato LJB, Vickers FF, et al. The effect of diclofenac sodium ophthalmic solution on intraocular pressure following cataract extraction. Ophthalmic Surg 1992;23:170-4.

20. Flach AJ, Stegman RC, Graham J, Kraper LP. Prophylaxis of aphakic cystoid macular oedema without corticosteroids: a paired-comparison, placebo-controlled double masked study. Ophthalmology 1990;97:1253-8.

21. Solomon KD. Efficacy of topical flurbiprofen and indomethacin in preventing pseudophakic cystoid macular oedema. Flurbiprofen Study Group I. J Cataract Refract Surg 1995;21:73-81.

22. Nishi O. Effect of intraocular sustained release of indomethacin on postoperative inflammation and posterior capsule opacification. J Cataract Refract Surg 1996;22(Suppl):806-10. 